\title{
Localized-density-matrix method and nonlinear optical response
}

\author{
WanZhen Liang, Satoshi Yokojima, and GuanHua Chen ${ }^{\text {a) }}$ \\ Department of Chemistry, The University of Hong Kong, Pokfulam Road, Hong Kong
}

(Received 22 November 1999; accepted 27 April 2000)

\begin{abstract}
The linear scaling localized-density-matrix (LDM) method is generalized to calculate the nonlinear optical responses of large polyacetylene oligomers. The ground state reduced single-electron density matrix is initialized by the divide-and-conquer approach and is obtained subsequently using the LDM method. The third-harmonic generation spectra of large oligomers are calculated. The saturation of off-resonant second hyperpolarizability $\chi^{(3)}(0)$ has been reinvestigated, and the value of $\chi^{(3)}(0)$ is found to depend mainly on the optical gap and the number of double bonds. (C) 2000 American Institute of Physics. [S0021-9606(00)30528-1]
\end{abstract}

\section{INTRODUCTION}

Recently a linear-scaling localized-density-matrix (LDM) method has been developed to evaluate the properties of excited states of very large electronic systems. ${ }^{1-7}$ It is based on the time-dependent Hartree-Fock (TDHF) approximation, ${ }^{8}$ which includes all single electron excitations and partial double, triple, and other multiple electron excitations. Instead of many-body wave functions, the reduced singleelectron density matrix $\rho$ is calculated from which physical observables such as the charge distribution, dipole moment, and photoexcitation spectrum are determined. Since the expensive calculation of many-body wave function is avoided, the computational cost decreases substantially. The reduced density matrix $\rho$ is described by an equation of motion

$$
i \hbar \dot{\rho}(t)=\langle[H, \rho]\rangle,
$$

and is obtained by direct integration in the time domain. Here $\rho \equiv\langle\hat{\rho}\rangle, \hat{\rho}$ is the reduced density matrix operator, and $H$ is the Hamiltonian. A truncation of $\rho$ is adopted for its elements when the distance between the two atomic orbitals involved is beyond a critical length. This truncation reduces the number of reduced density matrix elements to be determined from $N^{2}$ to $O(N)$ where $N$ is the number of the atomic orbitals in the system of interest, and leads subsequently to the linear scaling of the computational time versus the system size. The LDM method has so far been limited to calculating linear optical response, and has been applied to polyacetylene oligomers, carbon nanotubes, and poly( $p$ phenylenevinylene) aggregates. ${ }^{1-7}$

Nonlinear optical spectroscopy provides much more information than linear spectroscopy about the dynamic microscopic processes in the systems. For instance, time-resolved ultrafast spectroscopy reveals the breaking or forming of a chemical bond during a reaction; and the hole burning spectrum measures the inhomogeneity of a molecular system. Linear scaling computation for static nonlinear response has been reported, ${ }^{9,10}$ while it is still lacking for a dynamic nonlinear response. The LDM method has been applied to cal-

\footnotetext{
a) Author to whom all correspondence should be sent; electronic mail: ghc@yangtze.hku.hk
}

culate the linear response of very large electronic systems containing hundreds or thousands of atoms. ${ }^{1-7}$ It is desirable to generate it for calculating nonlinear response of very large and complex systems.

In this work we extend the LDM method to simulate nonlinear optical response. As the first application we study the third-harmonic generation (THG) spectra of large polyacetylene oligomers. The THG is one of most studied nonlinear optical processes. In Sec. II, the LDM method is briefly described and its generalization for calculating nonlinear optical response is described. The calculation procedure is outlined as well, for instance, the determination of initial and final ground state reduced single-electron density matrices and the selection of incident pulse. In Sec. III, the THG spectrum $\chi^{(3)}(-3 \omega ; \omega, \omega, \omega)$ is presented. The saturation of the off-resonant second hyperpolarizability $\chi^{(3)}(0)$ is examined. Finally, a discussion and conclusions are given in Sec. IV.

\section{FORMALISM}

Semiempirical models have been employed in the LDM method. ${ }^{1-7}$ These semiempirical models, such as the Pariser-Parr-Pople (PPP) model, ${ }^{11}$ the intermediate neglect of differential overlap (INDO), ${ }^{12}$ the modified neglect of diatomic overlap (MDNO) ${ }^{13}$ Austin Model 1 (AM1), ${ }^{14}$ and MNDO-Parametic Method 3 (PM3) ${ }^{15}$ consider only the valence electrons and neglect the differential overlaps for atomic orbitals on the same or different atoms. The semiempirical Hamiltonian in the presence of an external field $\mathbf{E}$ is described as follows:

$$
H=\sum_{m n} t_{m n} c_{m}^{\dagger} c_{n}+\frac{1}{2} \sum_{m n, i j} V_{m n, i j} c_{m}^{\dagger} c_{i}^{\dagger} c_{j} c_{n}-\mathbf{E}(t) \cdot \hat{\mathbf{P}},
$$

where $c_{m}^{\dagger}\left(c_{n}\right)$ is the creation (annihilation) operator for an electron at the localized atomic orbital $m(n) . t_{m n}$ is oneelectron integral of the atomic orbitals $m$ and $n$. When $m$ $=n, t_{m m}$ is the energy of the atomic orbital $m$; and when $m \neq n, t_{m n}$ is the hopping matrix element for $m$ and $n . V_{m n, i j}$ is the two-electron Coulomb interaction of the atomic orbital $m$ and $n$ on atom 1 and $i$ th and $j$ th atomic orbital on atom 2 . $-\mathbf{E}(t) \cdot \hat{\mathbf{P}}$ denotes the dipole interaction between the elec- 
trons and an external electric field $\mathbf{E}(t)$, where $\hat{\mathbf{P}}$ is the molecular polarization operator of the system. The electronic polarization can be expressed as

$$
\mathbf{P}(t)=\langle\Phi(t)|\hat{\mathbf{P}}| \Phi(t)\rangle,
$$

where $\Phi(t)$ is the wave function of the system. $\mathbf{P}$ may be expanded in powers of external field as

$$
\begin{aligned}
\mathbf{P} & =\mathbf{P}^{(0)}+\mathbf{P}^{(1)}+\mathbf{P}^{(2)}+\mathbf{P}^{(3)}+\cdots \\
& =\mathbf{P}^{(0)}+\chi^{(1)}: \mathbf{E}+\chi^{(2)}: \mathbf{E}^{2}+\chi^{(3)}: \mathbf{E}^{3}+\cdots
\end{aligned}
$$

with the $n$th order polarization $(n=1,2,3, \ldots)$

$$
\mathbf{P}^{(\mathbf{n})}(t)=\operatorname{Tr}\left(\hat{\mathbf{P}} \delta \rho^{(n)}\right),
$$

where the trace $\operatorname{Tr}$ is over all the atomic orbitals. $\mathbf{P}^{(1)}$ is the linear polarization, $\mathbf{P}^{(2)}, \mathbf{P}^{(3)}$ are the second- and third-order polarizations, respectively. $\chi^{(1)}, \chi^{(2)}, \chi^{(3)}$ are the first-, second-, and third-order polarizabilities, respectively. $\delta \rho^{(n)}$ is the $n$ th-order induced density matrix in the external field.

Consider a closed shell system containing $2 n$ electrons that occupy $n$ spatial molecular orbitals. The ground state wave function may be approximated by a singlet Slater determinant. The spin indices are omitted because of the symmetry between spin up and spin down. As a consequence, the ground state Fock matrix $h^{(0)}$ may be written as

$$
h_{m n}^{(0)}=t_{m n}+2 \sum_{i j} \rho_{i j}^{(0)} V_{m n, i j}-\sum_{i j} \rho_{i j}^{(0)} V_{m i, j n},
$$

where $\rho^{(0)}$ is the Hartree-Fock ground state reduced density matrix. Similarly, the induced Fock matrix $\delta h$ may be expressed as

$$
\delta h_{m n}=2 \sum_{i j} \delta \rho_{i j} V_{m n, i j}-\sum_{i j} \delta \rho_{i j} V_{m i, j n},
$$

where $\delta \rho$ is the induced reduced density matrix, $\delta \rho=\delta \rho^{(1)}$ $+\delta \rho^{(2)}+\delta \rho^{(3)}+\cdots$.

Within the TDHF approximation, the reduced singleelectron density matrix satisfies the following equation of motion:

$$
i \hbar \frac{d}{d t} \rho(t)=[h(t)+f(t), \rho(t)],
$$

where $h(t)=h^{(0)}+\delta h(t)$ is the Fock matrix, and $f(t)$ describes the interaction between an electron and the external field $\mathbf{E}(t),{ }^{16-18}$

$$
f_{m n}(t)=-\mathbf{E}(t) \cdot \hat{\mu}_{m n},
$$

where $\hat{\mu}_{m n}$ is the dipole moment element.

$\rho$ and $h$ may be written as

$$
\begin{aligned}
& \rho=\rho^{(0)}+\delta \rho^{(1)}+\delta \rho^{(2)}+\delta \rho^{(3)}+\cdots, \\
& h=h^{(0)}+\delta h^{(1)}+\delta h^{(2)}+\delta h^{(3)}+\cdots,
\end{aligned}
$$

respectively. $\delta h^{(n)}$ are the $n$th order induced Fock matrix in $\mathbf{E}(t)$. Equation (8) may thus be rewritten as

$$
i \hbar \frac{d}{d t} \delta \rho^{(1)}=\left[h^{(0)}, \delta \rho^{(1)}\right]+\left[\delta h^{(1)}, \rho^{(0)}\right]+\left[f, \rho^{(0)}\right],
$$

$$
\begin{aligned}
i \hbar \frac{d}{d t} \delta \rho^{(2)}= & {\left[h^{(0)}, \delta \rho^{(2)}\right]+\left[\delta h^{(2)}, \rho^{(0)}\right] } \\
& +\left[\delta h^{(1)}, \delta \rho^{(1)}\right]+\left[f, \rho^{(1)}\right], \\
i \hbar \frac{d}{d t} \delta \rho^{(3)}= & {\left[h^{(0)}, \delta \rho^{(3)}\right]+\left[\delta h^{(3)}, \rho^{(0)}\right]+\left[\delta h^{(2)}, \delta \rho^{(1)}\right] } \\
& +\left[\delta h^{(1)}, \delta \rho^{(2)}\right]+\left[f, \rho^{(2)}\right] .
\end{aligned}
$$

Equations (11)-(13) may be solved in time domain. ${ }^{19}$

The external field is expressed as

$$
\mathbf{E}(t)=\mathcal{E}(t) e^{\mathbf{i k} \cdot \mathbf{r}-i \omega_{0} t},
$$

where $\mathcal{E}(t)$ denotes the laser-pulse shape function, $\omega_{0}$ is the center frequency of the laser, and $\mathbf{k}$ is the propagation wave vector. We set $\mathcal{E}(t)=(1 / \sqrt{\pi} t) e^{-(t / \bar{t})^{2}} \hat{e}_{z}$, which is polarized along the $\hat{e}_{z}$ direction, where $\bar{t}$ denotes the pulse duration. The rotating frame is introduced to integrate Eqs. (11), (12), and (13), i.e., $\delta \rho^{(n)} \equiv \delta \bar{\rho}^{(n)} e^{-i n \omega_{0} t}, \delta h^{(n)} \equiv \delta \bar{h}^{(n)} e^{-i n \omega_{0} t}$, and $f \equiv \bar{f} e^{-i \omega_{0} t}$, and thus

$i\left(\hbar \frac{d}{d t}-i \omega_{0}\right) \delta \bar{\rho}^{(1)}=\left[h^{(0)}, \delta \bar{\rho}^{(1)}\right]+\left[\delta \bar{h}^{(1)}, \rho^{(0)}\right]+\left[\bar{f}, \rho^{(0)}\right]$,

$i\left(\hbar \frac{d}{d t}-2 i \omega_{0}\right) \delta \bar{\rho}^{(2)}=\left[h^{(0)}, \delta \bar{\rho}^{(2)}\right]+\left[\delta \bar{h}^{(2)}, \rho^{(0)}\right]$

$$
+\left[\delta \bar{h}^{(1)}, \delta \bar{\rho}^{(1)}\right]+\left[\bar{f}, \bar{\rho}^{(1)}\right]
$$

$$
\begin{aligned}
i\left(\hbar \frac{d}{d t}-3 i \omega_{0}\right) \delta \bar{\rho}^{(3)}= & {\left[h^{(0)}, \delta \bar{\rho}^{(3)}\right]+\left[\delta \bar{h}^{(3)}, \rho^{(0)}\right] } \\
& +\left[\delta \bar{h}^{(2)}, \delta \bar{\rho}^{(1)}\right]+\left[\delta \bar{h}^{(1)}, \delta \bar{\rho}^{(2)}\right] \\
& +\left[\bar{f}, \bar{\rho}^{(2)}\right] .
\end{aligned}
$$

To obtain the THG spectrum, we solve successively Eqs. (15) -(17) in time domain.

The third-order polarization $P^{(3)}(t)$ is related to the second hyperpolarizability $\chi^{(3)}$ as

$$
\begin{aligned}
P^{(3)}(t)= & \int d \omega_{1} d \omega_{2} d \omega_{3} \chi^{(3)}\left(-\omega_{1}-\omega_{2}-\omega_{3} ; \omega_{1}, \omega_{2}, \omega_{3}\right) \\
& \times E\left(\omega_{1}\right) E\left(\omega_{2}\right) E\left(\omega_{3}\right) e^{-i\left(\omega_{1}+\omega_{2}+\omega_{3}\right) t} \\
\approx & \chi^{(3)}\left(-3 \omega_{0} ; \omega_{0}, \omega_{0}, \omega_{0}\right) E^{3}\left(\omega_{0}\right) e^{-3 i \omega_{0} t}
\end{aligned}
$$

Thus the third-order polarization in frequency domain $P^{(3)}(3 \omega)$ may be evaluated as

$$
\begin{aligned}
P^{(3)}(3 \omega) & =\int d t P^{(3)}(t) e^{3 i \omega t} \\
& \approx \chi^{(3)}\left(-3 \omega_{0} ; \omega_{0}, \omega_{0}, \omega_{0}\right) E^{3}\left(\omega_{0}\right) \delta\left(\omega-\omega_{0}\right) .
\end{aligned}
$$

Therefore, for larger $\bar{t}$, the second hyperpolarizability

$$
\chi^{(3)}\left(-3 \omega_{0} ; \omega_{0}, \omega_{0}, \omega_{0}\right) \approx P^{(3)}\left(3 \omega_{0}\right) / E^{3}\left(\omega_{0}\right) .
$$

The fourth-order Runge-Kutta method ${ }^{20}$ is used to integrate Eqs. (15)-(17). The following approximations 
(a) $\rho_{i j}^{(0)}=0$ if $r_{i j}>l_{0}$,

(b) $\delta \rho_{i j}^{(n)}=0$ if $r_{i j}>l_{n}$,

are employed, ${ }^{1-7}$ which lead directly to

(c) $h_{i j}^{(0)}=0$ if $r_{i j}>l_{0}$,

(d) $\delta h_{i j}^{(n)}=0$ if $r_{i j}>l_{n}$.

With (a)-(d), the range of the summation $\Sigma_{k}$ is limited to a finite region for each term on the right-hand side of Eqs. (15)-(17). Thus, the computational cost for each $\delta \rho_{i j}^{(n)}$ is finite (i.e., not depending on $N$ ). Since only $O(N)$ number of $\delta \rho_{i j}^{(n)}$ are to be determined [because of (b)], the total number of computational steps are then $O(N)$ as well. The fast multipole method (FMM) ${ }^{21-24}$ or the cell multipole method ${ }^{25,26}$ is employed to calculate $h_{k k}^{(0)}$ and $\delta h_{k k}^{(n)}$. This ensures the finite computational time for each $h_{k k}^{(0)}$ and $\delta h_{k k}^{(n)}$ and thus guarantees that the total computational time scales linearly with the system size. Different orders of density matrix in $\mathbf{E}(t)$ have different critical lengths. Usually the higher the order of response $n$ is, the longer the critical length $l_{n}$ is, i.e., $l_{0} \leqslant l_{1} \leqslant l_{2} \leqslant l_{3} \leqslant \cdots$.

The Hartree-Fock ground state density matrix $\rho^{(0)}$ is also obtained via the linear scaling LDM calculation, ${ }^{4}$ and is determined by

$$
\left[h^{(0)}, \rho^{(0)}\right]=0
$$

together with the idempotency condition $\rho^{(0)} \rho^{(0)}=\rho^{(0)}$. The TDHF equation (8) is employed to determine $\rho^{(0)}$ by setting $\mathbf{E}(t)=0$ or $f(t)=0 .{ }^{4}$ Starting from an initial guess $\rho\left(t_{0}\right)$ for density matrix at time $t=t_{0}$, we follow its time evolution by integrating

$$
i\left(\hbar \frac{d}{d t}+\gamma^{\prime}\right) \rho(t)=[h(t), \rho(t)],
$$

while $\gamma^{\prime} \equiv \gamma^{\prime}(t)$ is time dependent, approaches zero over the time, and thus leads to the eventual convergence of $\rho(t)$ to $\rho^{(0)}$. The idempotency condition is then imposed by applying repeatedly $\rho^{\prime}=3(\rho)^{2}-2(\rho)^{3}$ until a convergence is reached. ${ }^{27,28}$ The resulting $\rho^{\prime}$ is then taken as the new trial density matrix. The above-mentioned process is applied iteratively until the density matrix $\rho(t)$ is converged. ${ }^{3,4}$ The initial guess $\rho\left(t_{0}\right)$ is preferably close enough to the real ground state density matrix $\rho^{(0)}$, and is obtained by the divide-and-conquer approach. ${ }^{29}$

\section{APPLICATION TO POLYACETYLENE}

A polyacetylene (PA) oligomer is a planar $\pi$-conjugated molecule, and its valence molecular orbitals (MOs) may be divided into $\pi$ and $\sigma$ MOs. ${ }^{11}$ It has been pointed out that the $\pi$ electrons are mainly responsible for the response in the optical range. ${ }^{30-33}$ The Pariser-Parr-Pople (PPP) Hamiltonian is employed to describe the dynamics of $\pi$ electrons. The zero differential overlap approximation ${ }^{34} V_{m i, n j}$ $=\delta_{m i} \delta_{n j} V_{m n}$ is adopted in the PPP Hamiltonian. The effective Coulomb interaction between two $\pi$ electrons may be expressed by the Ohno formula ${ }^{35}$

$$
V_{m n}=\frac{U_{0} / \epsilon}{\sqrt{1+\left(r_{m n} / a_{0}\right)^{2}}},
$$

\begin{tabular}{|c|c|c|c|c|c|}
\hline \multirow[b]{2}{*}{ Method } & \multicolumn{2}{|c|}{ Bond length $(\AA)$} & \multirow{2}{*}{$\begin{array}{c}\text { Bond angle } \\
\text { (deg) }\end{array}$} & \multirow[b]{2}{*}{$\Delta$} & \multirow{2}{*}{$\begin{array}{c}\beta^{\prime} \\
(\mathrm{eV} / \AA)\end{array}$} \\
\hline & Double bond & Single bond & & & \\
\hline Gaussian & 1.3371 & 1.4523 & 124.33 & 0.050935 & -3.56 \\
\hline AM1 & 1.3470 & 1.4436 & 122.89 & 0.042255 & -4.40 \\
\hline PM3 & 1.3443 & 1.4490 & 121.88 & 0.04576 & -4.00 \\
\hline
\end{tabular}

TABLE I. The optimized geometries of polyacetylene oligomers.

where $r_{m n}$ is the distance between the two orbitals $m$ and $n$, and $U_{0} / \epsilon$ is the on-site Coulomb repulsion. $\epsilon$ is the static dielectric constant caused by the polarization of core and $\sigma$ electrons. $a_{0}$ is of the same magnitude as the bond length. $U_{0}=11.13 \mathrm{eV}, \epsilon=1.5$, and $a_{0}=1.2935 \AA$ are used in the calculation.

The on-site energy $t_{m m}$ and the hopping matrix elements $t_{m n}$ other than the nearest neighbors are set to zero. The nearest-neighbor hopping matrix elements $t_{m, m \pm 1}$ or $t_{m \pm 1, m}$ are expressed as

$$
t_{m, m \pm 1}=\beta_{0}+(-1)^{n} \beta^{\prime} \Delta,
$$

as in Refs. 16-18. $t_{m, m \pm 1}$ depends on the bond-length alternation parameter $\Delta .2 \Delta$ is the $z$ component of the average bond length difference for single and double bonds. The PA chain is aligned along the $z$ axis. $\beta_{0}$ is set to $2.4 \mathrm{eV}$, the $\beta^{\prime}$ value is listed in Table I for different optimized structures. The electric field is polarized along the $z$ axis. The duration $\bar{t}$ of the stimulating electric pulse is set to $30 \mathrm{fs}$. The simulation time period $t$ is from -90.0 to 125.0 fs for each frequency $\omega_{0}$. The time step $\Delta t=0.1 \mathrm{fs}$ is employed. When the duration of laser pulse $\bar{t}$ is increased from $30 \mathrm{fs}$ to $50 \mathrm{fs}$, little change is observed. Thus, $\bar{t}=30 \mathrm{fs}$ is used for calculating the THG spectrum. The absorption spectrum is obtained with $\omega_{0}=0.0 \mathrm{eV}$ and $\bar{t}=0.1 \mathrm{fs}$, the simulation time from -0.5 to $70 \mathrm{fs}$, and time step $0.01 \mathrm{fs}$ is employed. In the calculation, the structures of large PA oligomers $(N>40)$ are based on the optimized geometry for $N=40$. The structure of the PA oligomer for $N=40$ is optimized using different methods, $a b$ initio Hartree-Fock methods with 6-31G basis set with GAUSSIAN $94,{ }^{36}$ or semiempirical methods like PM3 ${ }^{15}$ and AM1. ${ }^{14}$ The self-consistent convergence criteria $10^{-12}$ is used for the semiempirical calculations.

Figure 1 shows the linear absorption and THG spectra for $N=200(\sim 246 \AA)$ calculated by the LDM method. The cutoff lengths $l_{0}=l_{1}=50 \AA$ and $l_{2}=l_{3}=96 \AA$ are used in the calculation of THG spectra while $l_{0}=l_{1}=50 \AA$ are employed for the absorption. The phenomenological dephasing constant $\gamma$ is set to $0.1 \mathrm{eV}$. The structure is optimized by the $a b$ initio Hartree-Fock method. A three-level hierarchy is used in the FMM calculation with 25 atoms in the smallest box. The linear spectrum is plotted in Fig. 1(a). The inset shows the absorption spectrum for $N=200$ from $\omega=3.0$ to $5.5 \mathrm{eV}$. The amplitude has been magnified 105 times for clarity. The peak $a$ in $\left|\chi^{(3)}(-3 \omega ; \omega, \omega, \omega)\right|$ spectrum at $\omega=E_{g} / 3=0.67$ $\mathrm{eV}$, where $E_{g}$ is the optical gap, is attributed to the threephoton resonances and is consistent with the free-electron laser experimental result. ${ }^{37}$ Note that the resonance enhancements in $\left|\chi^{(3)}(-3 \omega ; \omega, \omega, \omega)\right|$ occur at $\omega=1.38,1.52$, and 

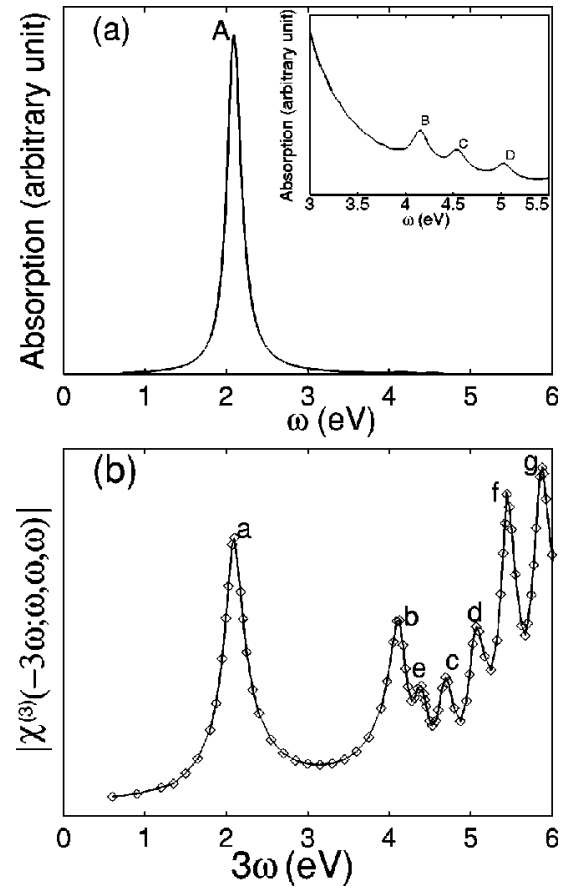

FIG. 1. (a) The optical absorption spectrum of PA with $N=200$. (b) The frequency-dependent second hyperpolarizability $\chi^{(3)}(-3 \omega ; \omega, \omega, \omega)$ for $N$ $=200$. The diamonds are the calculated results and the line provides guidance for the eyes in (b). Arbitrary units are used for absorption and THG.

$1.69 \mathrm{eV}\left[b, c\right.$, and $d$ in Fig. 1(b)] corresponding to $B_{u}$ states at $4.15,4.55$ and $5.06 \mathrm{eV}(B, C$, and $D)$ in the absorption spectrum, respectively. The peaks $e, f$, and $g$ are from $A_{g}$ states which do not appear in the absorption spectrum. The precise correspondence between $A$ and $a, B$ and $b, C$ and $c$, and $D$ and $d$ demonstrates the validity of the LDM calculation for nonlinear optical response. $l_{2}=l_{3}=96 \AA$ are thus employed in the rest of calculation for the THG.

In order to verify that the computational time scales linearly, the CPU time for calculating $\chi^{(3)}(0)$ is recorded for different values of $N$. The critical lengths $l_{0}=l_{1}=l_{2}=l_{3}$ $=96 \AA$ are employed and 12 atoms in the smallest box are included for the FMM calculation. Each calculation is performed during a time interval $[-90 \mathrm{fs},-88 \mathrm{fs}]$ with a time step $0.1 \mathrm{fs}$. The calculation is done by the $400 \mathrm{MHz}$ Pentium II computer with RedHat LINUX 5.2. The results are plotted in Fig. 2. Clearly, the CPU time is proportional to the system size $N$. Thus, the linear-scaling property of the LDM computational time is preserved for the nonlinear optical response calculation.

Saturation of the off-resonant second hyperpolarizability $\chi^{(3)}(0)$ has been investigated intensively. The saturation length varies from 40 to $200 \AA .{ }^{16,17}$ We examine this issue here. (For hyperpolarizability related issues, see e.g., Refs. 30,38 , and 39. For recent progress of the hyperpolarizability of PA, see Refs. 40-47.) The linear and third-order offresonant polarizabilities versus the size $N$ are plotted in Fig. 3 . To ensure the validity of the results, different geometries are employed. Ab initio Hartree-Fock, PM3, AM1 are used to obtain the optimized geometries for each oligomer. The solid, dotted, and dashed lines are the results for $\beta^{\prime}=3.56$ $\mathrm{eV} / \AA$. The $\Delta$ values are $0.051,0.046$, and $0.042 \AA$ for $a b$

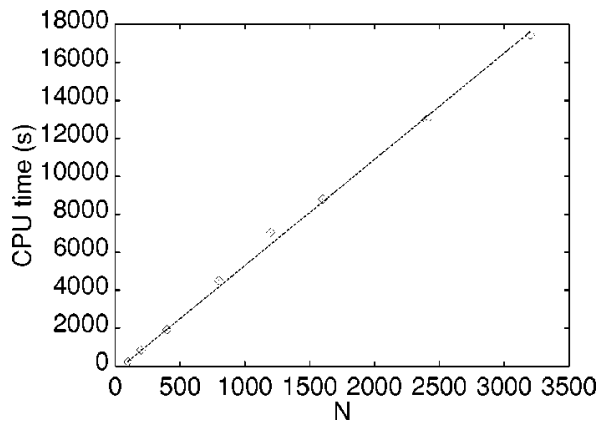

FIG. 2. The CPU time for the off-resonant second hyperpolarizability $\chi^{(3)}(0)$. The critical lengths $l_{0}=l_{1}=l_{2}=l_{3}=96 \AA$ are employed and 12 atoms in the smallest box are included. Each calculation is performed during a time interval $[-90 \mathrm{fs},-88 \mathrm{fs}]$ with a time step $0.1 \mathrm{fs}$.

initio, PM3, and AM1 optimized geometries, respectively. The saturation lengths are about 370,490 , and $610 \AA$ for the above-mentioned structures, respectively. The larger $\Delta$, is the shorter the saturation length. With $\beta^{\prime}=3.56 \mathrm{eV} / \AA$, the optical gaps are $2.0,1.92$, and $1.89 \mathrm{eV}$ for the three structures, respectively. Since the experimental value for the PA's optical gap is $2.0 \mathrm{eV}$, we adjust $\beta^{\prime}$ for the PM3 and AM1 optimized geometries so that the calculated gaps are equal to $2.0 \mathrm{eV}$. The resulting values are 4.0 and $4.4 \mathrm{eV} / \AA$, respectively. $\chi^{(3)}(0)$ is evaluated again, and the results are plotted against $N$ in Fig. 3(b). The results are very close to those for the $a b$ initio Hartree-Fock optimized geometry. In other words, the value of $\chi^{(3)}(0)$ is mainly determined by the op-
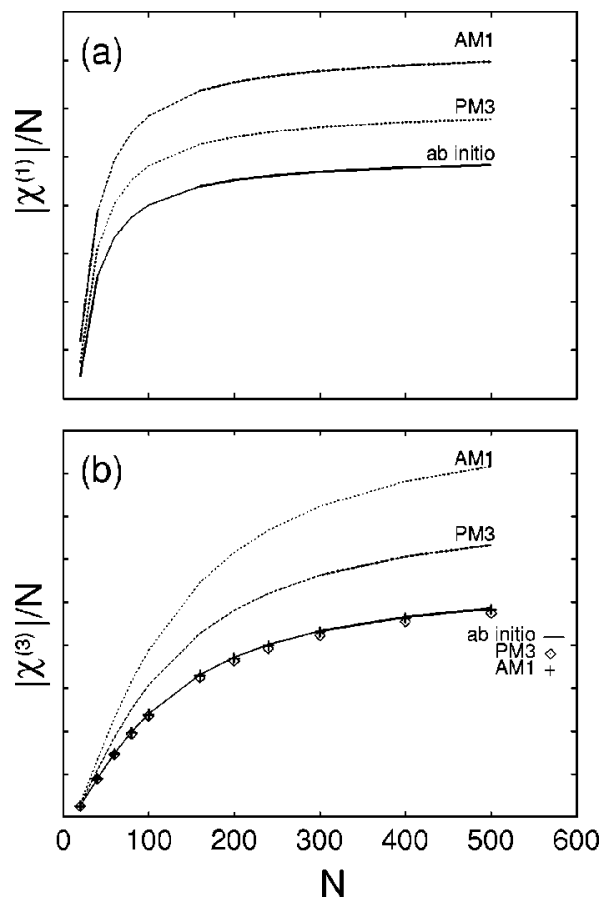

FIG. 3. The off-resonant optical polarizabilities vs $N$ for different optimized geometries. (a) The scaled off-resonant linear polarizability $\left|\chi^{(1)}\right| / N$ vs $N$ with $\beta^{\prime}=3.56 \mathrm{eV} / \AA$. (b) The scaled off-resonant second hyperpolarizability $\left|\chi^{(3)}(0)\right| / N$ vs $N . \beta^{\prime}=3.56 \mathrm{eV} / \AA$ for all lines. The solid, dotted, and dashed lines correspond to the optimized geometries obtained by ab initio HartreeFock, PM3 and AM1, respectively. The $\beta^{\prime}$ values for the crosses and diamonds in (b) are 4.4 and $4.0 \mathrm{eV} / \AA$, respectively. The crosses and diamonds are for AM1 and PM3 optimized structures, respectively. 
tical gap and $N$, and is insensitive to small variation of the bond length alternation $\Delta$. The onset of the saturation of $\left|\chi^{(3)}\right|$ occurs at about $N=300$ carbon atoms. It is consistent with the result of a recent experiment ${ }^{48,49}$ and agrees with a numerical calculation by Mukamel and co-workers. ${ }^{17}$ On the other hand, the saturation length for $\chi^{(1)}(0)$ is about $N$ $=100$ [see Fig 3(a)], which is consistent with the finding of Ref. 16.

\section{CONCLUSION}

The linear-scaling method, for instance, the divided-andconquer method, ${ }^{29}$ and the density-matrix minimization approach ${ }^{28}$ have been used to calculate the ground state energy and charge distribution. Usually a cutoff length of $10 \AA$ is adopted. The resulting reduced density matrix yields accurate energy and charge distribution for the ground state. However, it might yield unphysical features in the absorption spectrum if the reduced ground state density matrix obtained with the short cutoff length is employed directly for the excited state property calculation. Thus, it is essential that a large cutoff length is used for the ground state calculation to ensure the validity and accuracy of subsequent excited state property calculation. In our calculation, the cutoff length $l_{0}$ $=50 \AA$ is used to obtain the reduced ground state density matrix.

The computational time required for a LDM calculation scales also as $O\left(M^{3}\right)$, where $M$ is the number of atomic orbitals within the critical length $l_{n}$. The larger the critical length $l_{n}$, the higher the computational cost. Since a larger value of $l_{3}$ is required for the THG calculation than $l_{1}$ for the absorption spectrum, the computational time for calculating the THG spectrum is higher than that of the linear response. In addition, one stimulating laser pulse centered around $\omega_{0}$ may only be used to calculate $\chi^{(3)}\left(-3 \omega_{0} ; \omega_{0}, \omega_{0}, \omega_{0}\right)$, one point in the THG spectrum. Thus, it is still relatively expensive to calculate the nonlinear optical response using the LDM method, although the computational time does scale linearly with the system size.

To summarize, we have generalized the linear-scaling LDM method to calculate the nonlinear optical response of very large systems. The computational time has been demonstrated to scale linearly with the system size for polyacetylene oligomers containing up to 3200 carbon atoms. The second hyperpolarizabilities of polyacetylene oligomers with up to 500 carbon atoms have been determined accurately. The values of off-resonant polarizabilities are found depending mainly on the optical gap and $N$ while small variation of geometry has little effect.

\section{ACKNOWLEDGMENTS}

Support from the Hong Kong Research Grant Council (RGC) and the Committee for Research and Conference Grants (CRCG) of the University of Hong Kong is gratefully acknowledged.

\footnotetext{
${ }^{1}$ S. Yokojima and G. H. Chen, Chem. Phys. Lett. 292, 379 (1998).

${ }^{2}$ S. Yokojima and G. H. Chen, Phys. Rev. B 59, 7259 (1999).

${ }^{3}$ S. Yokojima and G. H. Chen, Chem. Phys. Lett. 300, 540 (1999).
}

${ }^{4}$ S. Yokojima, D. H. Zhou, and G. H. Chen, Chem. Phys. Lett. 302, 495 (1999).

${ }^{5}$ W. Z. Liang, S. Yokojima, and G. H. Chen, J. Chem. Phys. 110, 1844 (1999).

${ }^{6}$ W. Z. Liang, S. Yokojima, D. H. Zhou, and G. H. Chen, J. Phys. Chem. A 104, 2445 (2000).

${ }^{7}$ S. Yokojima, X. J. Wang, D. H. Zhou, and G. H. Chen, J. Chem. Phys. 111, 10444 (1999).

${ }^{8}$ P. Ring and P. Schuck, The Nuclear Many-Body Problem (Springer, New York, 1980).

${ }^{9}$ R. W. Nunes and D. Vanderbilt, Phys. Rev. Lett. 73, 712 (1994).

${ }^{10}$ A. Dal Corso and F. Mauri, Phys. Rev. B 50, 5756 (1994).

${ }^{11}$ R. Pariser and R. G. Parr, J. Chem. Phys. 21, 767 (1953); J. A. Pople, Trans. Faraday Soc. 49, 1375 (1953).

${ }^{12}$ J. A. Pople, D. L. Beveridge, and P. A. Dobosh, J. Chem. Phys. 47, 2026 (1967).

${ }^{13}$ M. J. S. Dewar and W. Thiel, J. Am. Chem. Soc. 99, 4899 (1977).

${ }^{14}$ M. J. S. Dewar, E. G. Zoebisch, E. F. Healy, and J. J. P. Stewart, J. Am. Chem. Soc. 107, 3902 (1985).

${ }^{15}$ J. J. P. Stewart, J. Comput. Chem. 10, 209 (1989).

${ }^{16}$ S. Mukamel, A. Takahashi, H. X. Wang, and G. H. Chen, Science 266, 250 (1994).

${ }^{17}$ S. Tretiak, V. Chernyak, and S. Mukamel, Phys. Rev. Lett. 77, 4656 (1996).

${ }^{18}$ A. Takahashi and S. Mukamel, J. Chem. Phys. 100, 2366 (1994); G. H. Chen and S. Mukamel, J. Am. Chem. Soc. 117, 4945 (1995); S. Mukamel, Principles of Nonlinear Optical Spectroscopy (Oxford University Press, New York, 1995).

${ }^{19}$ T. Meier, S. Tretiak, V. Chernyak, and S. Mukamel, Phys. Rev. B 55, 4960 (1997)

${ }^{20}$ W. H. Press, B. P. Flannery, S. A. Teukolsky, and W. T. Vetterling, Numerical Recipes in C (Cambridge University Press, New York, 1988).

${ }^{21}$ L. Greengard and V. Rokhlin, J. Comput. Phys. 73, 325 (1987).

${ }^{22}$ L. Greengard, The Rapid Evaluation of Potential Fields in Particle Systems (MIT, Cambridge, MA, 1988).

${ }^{23}$ C. A. White, B. G. Johnson, P. M. W. Gill, and M. Head-Gordon, Chem. Phys. Lett. 253, 268 (1996).

${ }^{24}$ M. C. Strain, G. E. Scuseria, and M. J. Frisch, Science 271, 51 (1996).

${ }^{25}$ H.-Q. Ding, N. Karasawa, and W. A. Goddard III, Chem. Phys. Lett. 196, 6 (1992).

${ }^{26}$ H.-Q. Ding, N. Karasawa, and W. A. Goddard III, J. Chem. Phys. 97, 4309 (1992).

${ }^{27}$ R. McWeeny, Rev. Mod. Phys. 32, 335 (1960).

${ }^{28}$ X.-P. Li, R. W. Nunes, and D. Vanderbilt, Phys. Rev. B 47, 10891 (1993).

${ }^{29}$ W. Yang and T.-S. Lee, J. Chem. Phys. 103, 5674 (1995); T.-S. Lee, D. M. York, and W. Yang, J. Chem. Phys. 105, 2744 (1996).

${ }^{30}$ S. Etemad and Z. G. Soos, in Spectroscopy of Advanced Materials, edited by R. J. H. Clark and R. E. Hester (Wiley, New York, 1991), p. 87, and references therein.

${ }^{31}$ Z. G. Soos, S. Ramesesha, D. S. Galvao, and S. Etemad, Phys. Rev. B 47, 1742 (1993).

${ }^{32}$ I. Ohmine, M. Karplus, and K. Schulten, J. Chem. Phys. 68, 2298 (1978).

${ }^{33}$ H. Fukutome, J. Mol. Struct.: THEOCHEM 188, 337 (1989), and references therein.

${ }^{34}$ A. A. Hasanein and M. W. Evans, Computational Methods in Quantum Chemistry, Quantum Chemistry Vol. 2 (World Scientific, Singapore, 1996).

${ }^{35}$ K. Ohno, Theor. Chim. Acta 2, 219 (1964).

${ }^{36}$ M. J. Frisch et al., GAUSSIAN 94 (Revision C.3), Gaussian, Inc., Pittsburgh, PA, 1995.

${ }^{37}$ W. S. Fann, S. Benson, J. M. J. Madey, S. Etemad, G. L. Baker, and F. Kajzar, Phys. Rev. Lett. 62, 1492 (1989).

${ }^{38}$ S. P. Karna and A. T. Yeates, Nonlinear Optical Materials (American Chemical Society, Washington, DC, 1996), and references therein.

${ }^{39}$ E. K. Wilson, Chem. Eng. News 77, 29 (1999).

${ }^{40}$ S. R. Marder, J. W. Perry, G. Bourhill, C. B. Gorman, B. G. Tiemann, and K. Mansour, Science 261, 186 (1993).

${ }^{41}$ S. R. Marder, C. B. Gorman, F. Meyers, J. W. Perry, G. Bourhill, J.-L. Brédas, and B. M. Pierce, Science 265, 632 (1994). 
${ }^{42}$ M. Chandross, Y. Shimoi, and S. Mazumdar, Phys. Rev. B 59, 4822 (1999).

${ }^{43}$ R. J. Bursill and W. Barford, Phys. Rev. Lett. 82, 1514 (1999).

${ }^{44}$ Y. Luo, P. Norman, P. Macak, and H. Ågren, J. Chem. Phys. 111, 9853 (1999).

${ }^{45}$ A. Painelli, L. Del Freo, A. Girlando, and Z. G. Soos, Phys. Rev. B 60, 8129 (1999).
${ }^{46}$ M. Schulz, S. Tretiak, V. Chernyak, and S. Mukamel, J. Am. Chem. Soc. 122, 452 (2000).

${ }^{47}$ G. P. Zhang, Phys. Rev. B 61, 4377 (2000).

${ }^{48}$ I. D. W. Samuel, I. Ledoux, C. Dhenaut, J. Zyss, H. H. Fox, R. R. Schrock, and R. J. Silbey, Science 265, 1070 (1994).

${ }^{49}$ I. Ledoux, I. D. W. Samuel, J. Zyss, S. N. Yaliraki, F. J. Schattenmann, R. R. Schrock, and R. J. Silbey, Chem. Phys. 245, 1 (1999). 